\title{
STUDY OF EROSION-CORROSION OF STAINLESS STEEL, BRASS AND ALUMINUM BY OPEN CIRCUIT POTENTIAL MEASUREMENTS
}

\author{
Kazi Bayzid Kabir*, Iqbal Mahmud \\ Department of Chemical Engineering, Bangladesh University of Engineering and Technology (BUET), \\ Dhaka-1000, Bangladesh
}

\begin{abstract}
Influence of flow on corrosion behavior of stainless steel, brass and aluminum was studied using electrochemical measurement techniques. Flow effect was identified by comparing erosion-corrosion with pure corrosion studies. For this purpose, open circuit potential (OCP) measurements were performed in both quiescent and flow conditions. OCP transient for the metals/alloys (stainless steel, brass and aluminum) indicated the nature and extent of corrosion on the sample by the environment (seawater and sodium carbonate). Three distinct type of curves were found for all metal-environment combinations. Increasing OCP indicated noble metal behavior while decrease in $O C P$ indicated corrosion of material in the exposed environment. Directional shift in the OCP indicated changes in the surface phenomena. Hence, shape of these curves provides information about the extent of metal environment interaction. Experimental observations showed the applicability of open circuit measurements for on-line corrosion monitoring.
\end{abstract}

\section{Keywords}

open circuit potential; erosion-corrosion; electrochemical measurements; stainless steel; brass; aluminum.

\section{Introduction}

Relative movement between metals and environment has an effect on rate of corrosion, unless both anodic and cathodic processes are activation controlled ${ }^{1}$. Erosioncorrosion is the conjoint action involving corrosion and erosion in the presence of a moving corrosive fluid, leading to accelerated loss of material ${ }^{2}$. Erosion-corrosion can occur in different situations: in absence or presence of solids and gases. Hence, there is no widely acceptable mechanism of erosion-corrosion. However, there are suggestions that the corrosion process is enhanced by increased mass transfer and/or mechanical force impacts on the surface ${ }^{1,3}$. Therefore, models to predict erosioncorrosion are not readily available. Experimental studies are best way to predict occurrence of erosion-corrosion.

Electrochemical techniques provide a viable method for rapid prediction or evaluation of corrosion. Their applicability arises from the fact that most aqueous corrosion processes involving metals require the transfer of charge across the metal-solution interface. Electrochemical measurements in flowing solution can provide data on (a) the rate of general corrosion and the possibility of other forms of attack, (b) mechanism, (c) the characteristics hydrodynamic parameters, and (d) the

*Corresponding Author's Email : kazibayzid@che.buet.ac.bd composition of the solution by electro-analytically monitoring compositions or measuring red-ox potential, pH etc. ${ }^{4}$

Open circuit potential (OCP) is the electrode potential at which no net current flow through the external circuit of the electrochemical cell. OCP of a corroding metal/alloy is measured as voltage between the metal/alloy (working electrode) and a reference electrode using a highimpedance voltmeter. Measurement of OCP can provide:

- a method for determining whether the corrosion system is in the active or the passive state ${ }^{5}$.

- determination of the potential distribution on the corroding surface (heterogeneous mixed electrodes) ${ }^{6}$.

- data for corrosion monitoring in a plant or in the field ${ }^{7,8}$.

- information on the free corrosion potential as the starting point for the application of electrochemical protection methods

- data for control of the protection potential during electrochemical protection ${ }^{9}$.

This paper deals with the effect of flow on corrosion behavior of several metal/alloys, namely, stainless steel, brass and aluminum, in different corrosive environments 
by observation of open circuit potential. Results obtained for the stagnant conditions are compared with flow condition measurements.

\section{Experimental Details}

Closed-loop system designed by $\operatorname{Easin}^{10}$, after several modifications, was used for the current study. A schematic diagram of the test rig is shown in Fig. 1. Water is circulated from the reservoir by a centrifugal pump. Poly vinyl chloride (PVC) pipeline and fittings are used for water circulation to avoid corrosion which would be experienced with steel or copper tubes. The velocity of water from the nozzle can be adjusted by varying the opening of the bypass valve and geometry of the nozzle. The nozzle can be changed whenever required to vary the impact velocity. A rotameter is also installed to measure the water flow rate.

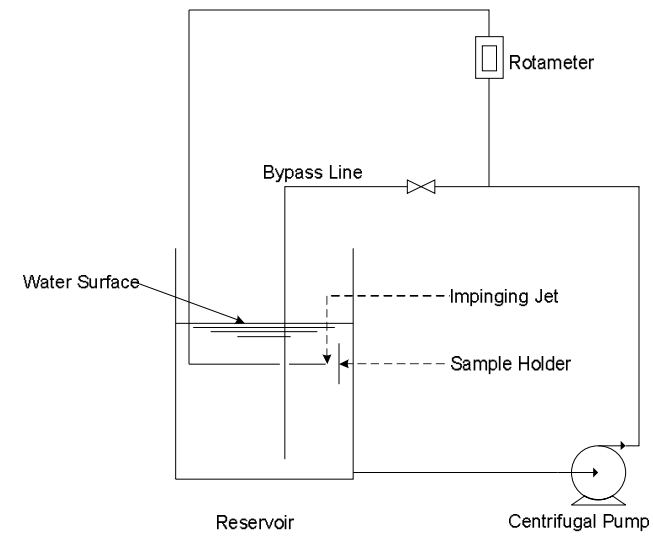

Fig 1. Erosion-Corrosion Test Rig with

The specimen holder, made of transparent Perspex sheet, can be rotated about its vertical axis. This enables to conduct impinging test runs for any impact angle between $0^{\circ}$ to $90^{\circ}$. The test rig can be used either as submerged jet or free jet. Lowering the water level in the reservoir below the impinging jet makes it a free jet.

Erosion-corrosion of stainless steel, brass and aluminum were investigated. Optical Emission Spectroscopy (OES) was used to determine compositions of the metal and alloys. Table 1 shows composition of the metal and alloys used.

Commercial grade $\mathrm{Na}_{2} \mathrm{CO}_{3}$ and raw sea salt were used for preparation of solutions. Commercial grades were selected since studies performed on analytical grades would be irrelevant for local industries using commercial grade chemicals only.

Rectangular working electrodes were made from commercial sheet of $1.5-\mathrm{mm}$ thickness. Since size of the specimen affects corrosion behavior11, 12, specimens were made sufficient large enough $(102 \mathrm{~mm} \times 76 \mathrm{~mm})$ to nullify the size effect. The prepared specimens were ground on 600 grit silicon carbide abrasive paper.

The specimens were then washed with distilled water, degreased with acetone and dried with hot air. The rear and the edges of the samples were coated with expanded polystyrene (EPS) solution in xylene to make sure that only the front section is exposed to the flow.

Table 1. Metal/alloy compositions

\begin{tabular}{|l|l|l|l|}
\hline Metal/alloy & Components & $\%$ & Designation \\
\hline Stainless steel & $\mathrm{Cr}$ & 17.187 & UNS \\
& $\mathrm{Ni}$ & 2.1167 & S20100 \\
& $\mathrm{C}$ & 0.1089 & \\
& $\mathrm{Mn}$ & 4.9511 & \\
& $\mathrm{Si}$ & 0.5759 & \\
& $\mathrm{P}$ & 0.0452 & \\
& $\mathrm{~S}$ & 0.0106 & \\
\hline Aluminum & $\mathrm{Al}$ & $>99$ & \\
\hline Brass & $\mathrm{Cu}$ & 62.753 & CW508L \\
& $\mathrm{Zn}$ & 36.972 & \\
\hline
\end{tabular}

A cell consisting of working electrode and a saturated calomel electrode (SCE) was formed for measuring equilibrium potentials. OCP of the formed cells were recorded by using a data acquisition system which was connected to a computer through RS-232 port.

\section{Results}

Fig. 2 shows OCP of SS201 in seawater as a function of time. Under fluid jet impingement, the potential increases very sharply immediately after exposing to the environment. After first $40 \mathrm{~s}$ the rate decreases and shows an exponential behavior until pitting potential of $187 \mathrm{mV}$ (at approximately $1200 \mathrm{~s}$; shown with an arrow in Fig 2) is reached. However, the potential then starts to decrease at a slow rate. The rate and fluctuations increases as the time goes on.

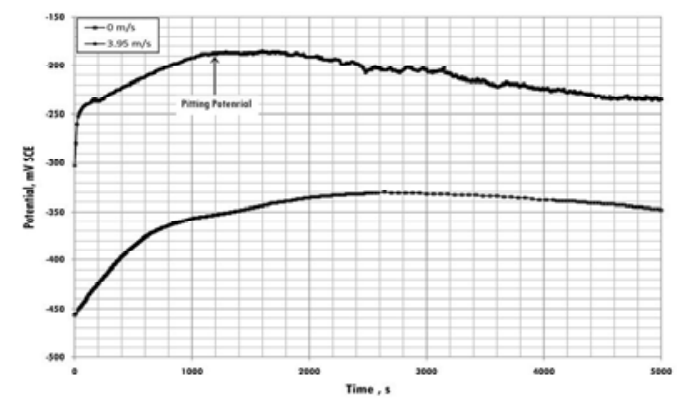

Fig 2. OCP transient of SS201 in seawater

OCP of SS201 in quiescent condition also increases with time. However, the rate is much slower than the flow conditions. The potential also does not reach the pitting potential within the experimental period of $5000 \mathrm{~s}$. At quiescent condition, the potential reaches a maximum of $-330 \mathrm{mV}$ at approximately $3000 \mathrm{~s}$ and then shows a very slow decline.

When exposed to $2.5 \% \mathrm{Na}_{2} \mathrm{CO}_{3}, \mathrm{SS} 201$ and brass show similar trend in OCP. SS201 starts with a potential 
difference of $-510 \mathrm{mV}$ with respect to SCE (Fig. 3). The potential then starts to increase. However, the rate starts to diminish with time and OCP settles to a value of -373 $\mathrm{mV}$ after $5000 \mathrm{~s}$. Under fluid impingement, initial potential shifts to a more positive direction, $-440 \mathrm{mV}$ and reaches to $-200 \mathrm{mV}$ after $5000 \mathrm{~s}$.

OCP of brass in seawater is shown in Fig. 4. In both quiescent and fluid impingement condition the potential change after the initial induction period is very slow leading to almost constant potential after a period of approximately $3500 \mathrm{~s}$. The shape of the curve indicates formation of protective layer on the surface. In flow condition, protective layer of the fluid impact zone continuously get destroyed followed by reformation of the layer.

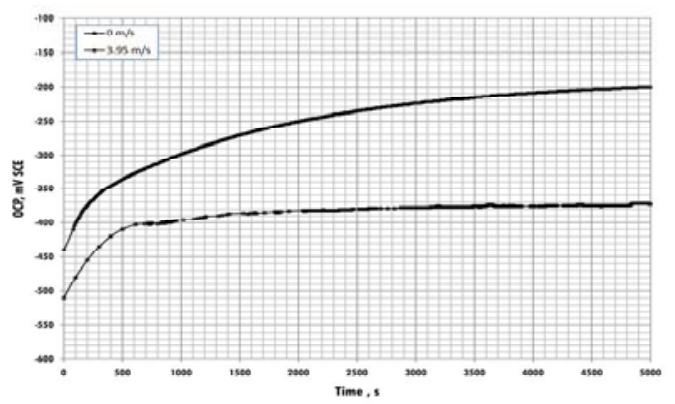

Fig 3. OCP transient of $\mathrm{SS}_{201}$ in $\mathrm{Na}_{2} \mathrm{CO}_{3}$

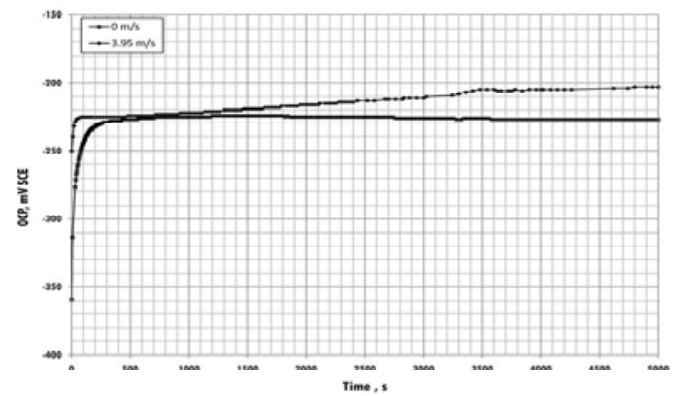

Fig 4. OCP transient of brass in seawater

Brass has initial OCP of $-505 \mathrm{mV}$ for no flow and -435 $\mathrm{mV}$ for impact velocity of $3.95 \mathrm{~m} / \mathrm{s}$ when exposed to $\mathrm{Na}_{2} \mathrm{CO}_{3}$. The OCP then increases and after an induction period of approximately $1000 \mathrm{~s}$ shows relatively small variation (Fig. 5).

OCP of aluminum in seawater in totally different from the other two specimens, as shown in Fig. 6. After exposing the aluminum specimen to artificial seawater the OCP starts to decrease. At quiescent condition, OCP decreases at a steady rate. But, at flow conditions, OCP of aluminum decreases at a very fast rate upto $200 \mathrm{~s}$. The rate, then becomes much slower. However, the curve shows large amount of oscillations indicating destruction and formation of corrosion product layer at the surface. The convex shape of OCP for aluminum indicates that it undergoes severe corrosion when exposed to seawater. Even at the end of a experimental period of $5000 \mathrm{~s}$ the rate of decline in OCP is quite considerable.

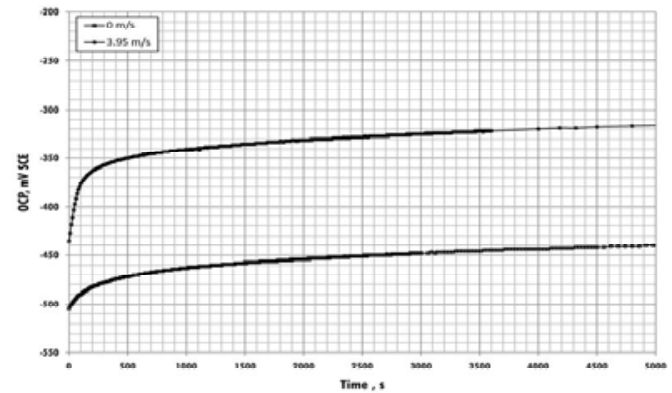

Fig 5. OCP transient of brass in $\mathrm{Na}_{2} \mathrm{CO}_{3}$

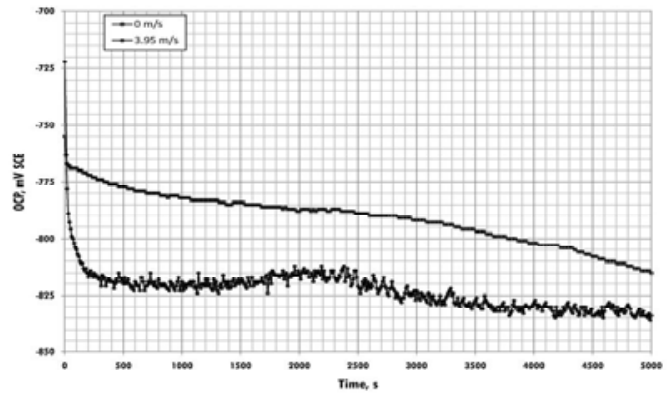

Fig 6. OCP transient of aluminum in seawater

Like seawater, behavior of aluminum in $\mathrm{Na}_{2} \mathrm{CO}_{3}$ is quite different from other two alloys as shown in Fig. 7. For quiescent condition, the OCP initially increases upto 400 $\mathrm{s}$. For next $400 \mathrm{~s}$ the potential remains within -1556 to $-1564 \mathrm{mV}$. After that the potential gradually decreases indicating significant attack on the metal and continues upto $1700 \mathrm{~s}$. This phase is followed by a steady value of OCP for rest of the experimental time. The metal gets anodized to give a protective layer and therefore OCP variation is insignificant here.

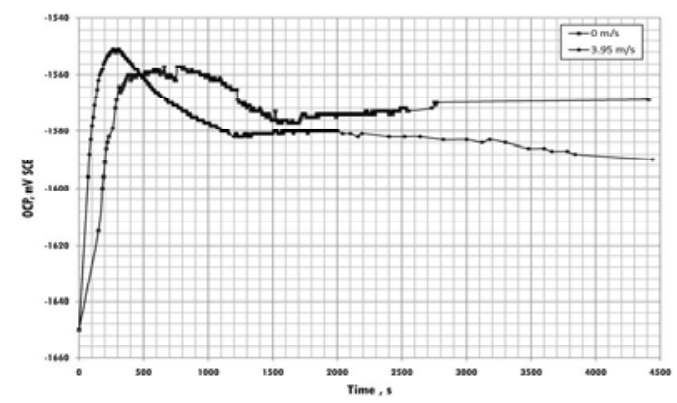

Fig 7. OCP transient of aluminum in $\mathrm{Na}_{2} \mathrm{CO}_{3}$

At flow condition, induction period is reached at a faster rate and only in $300 \mathrm{~s}$. Then the potential gradually decreases initially at faster rate. Rate of change in OCP slows down with time, but does not disappear completely. The anodizing effect is therefore absent in case of flow.

\section{Discussion}

Electrochemical measurements were made to study the effect of flow on corrosion. Tests were performed in the 
turbulent flow regime since erosion-corrosion occurs mostly under such conditions. All the experiments were performed under steady flow rates $(2.63 \mathrm{~m} / \mathrm{s}$ and 3.95 $\mathrm{m} / \mathrm{s}$ ) without any temperature control.

Reynolds number for an impinging jet is given by: $R e=$ $d u \rho / \mu$, where, $d$ is the diameter of the impinging nozzle, $u$ is the solution velocity, and $\rho$ and $\mu$ is the density and viscosity of the solution. For a flow velocity of $2.63 \mathrm{~m} / \mathrm{s}$ using a nozzle of 0.5 in diameter, $R e$ under flow impingement is calculated to be 333000 . Reynolds number is 500000 for flow velocity of $3.95 \mathrm{~m} / \mathrm{s}$ for the same system. This value indicates that the flow is turbulent since the critical value for laminar to turbulent flow in a impinging jet system is $2000^{4}$. Turbulent flow near the surface enhanced oxygen mass transfer rate to the electrode surface and reduced the thickness of the diffusion boundary layer. Therefore, flow increases oxygen reduction at the surface and also enhance the anodic reaction.

As soon as the sample specimens were immersed in the corroding environment OCP was found to vary with time. Variation of OCP depends on the metal/alloy composition, nature of the corrosive environment, flow conditions, temperatures etc. Depending on the conditions OCP can shift either in more noble or less noble direction or can be a combination of both.

While studying the OCP transients of the metal/alloys, three distinct types of potential-time curves are observed:

- Case A: Potential becomes more and more noble, the metal or alloy becomes protected by the oxide layer formation: brass and stainless steel in $\mathrm{Na}_{2} \mathrm{CO}_{3}$.

- Case B: Potential becomes noble and then starts to decrease. This is observed when the protective layer of the metal or alloy is modified by the environmental factors (e.g. breakdown of passivation of SS201 in presence of chloride).

- Case C: Potential becomes less and less noble, the metal or alloy severely attacked by the environment: aluminum in seawater.

Case A is caused by enhanced cathodic reaction (oxygen reduction) and enhanced anodic reaction (growth of the passive film) on the surface. In such cases, corrosion products are formed instantaneously as soon as the specimens are exposed to the corrosive environment. The potential starts to shift to the noble direction. After stable diffusion barrier is developed the material gets isolated from the environment and metal dissolution rate drops considerably. As a result insignificant changes in surface potential is observed.

Case B is observed for SS201 when exposed to flowing seawater. Initially the polarization shifts to the noble direction indicating formation of diffusion boundary layer on the metal surface. However, turbulent flow causes thinner diffusion boundary layer and enhanced oxygen transfer rate. The turbulent flow also washes away anolyte from the surface. This causes migration of more metal ions to the anolyte. Chloride ions then migrates to maintain the electroneutrality. Hence the anolyte has a high concentration of $\mathrm{FeCl}_{2}$ and thereby lowering the anolyte $\mathrm{pH}$. It is shown earlier that at a concentration higher than $3 \mathrm{M} \mathrm{FeCl}_{2}$ dissolution of stainless steel sustains in chloride medium ${ }^{13}$. When this concentration is obtained, pits start to form on the surface. The net effect is shift of polarization curve to the active direction. Formation of pit is followed by repassivation. This explains the oscillations in the potential in this region. However, this phenomena is not as severe as observed for SS under impingement by free jets ${ }^{13}$.

Similar curve is found for aluminum in $\mathrm{Na}_{2} \mathrm{CO}_{3}$. For aluminum, the potential shift in the negative reduction was observed after a certain period of time for both quiescent and flow conditions. Here the oxide film is dissolved by the alkaline dissolution of $\mathrm{Al}_{2} \mathrm{O}_{3}$ to $\mathrm{AlO}^{2-}$. The rate of dissolution increased in presence of flow and observed by the faster rate of potential shift in the active direction.

Case $\mathrm{C}$ is observed for aluminum in seawater. Aluminum is prone to pitting corrosion in presence of chlorides ions and at a $\mathrm{pH}$ close to neutrality ${ }^{14}$. The oxide layer dissolves in the solution in the form of $\mathrm{Al}^{3+}$ and does not act as effective boundary layer. More severe conditions are observed in turbulent flow conditions. The dissolution rate is increased by accelerated diffusion to and from the surface and the potential continues to decrease.

\section{Conclusion}

Erosion-corrosion of stainless steel, brass and aluminum was studied under impingement by a fluid jet. Submerged fluid jet was selected since it closely simulates fluid flow in process industries.

OCP measurements were made for the study. Shape of OCP transient showed the severity of attack on metal/alloy by the environment. Results obtained through OCP measurements also agree with those obtained by weight loss measurements. It is a simple and cheap technique and can be used as a tool for on-line monitoring of corrosion.

\section{Acknowledgement}

KBK would like to thank Dr. Md. Moniruzzaman of Materials and Metallurgical Engineering Department of Bangladesh University of Engineeing and Technology for technical and instrumental support during this research work.

\section{References}

1. Poulson, B. S., Corrosion. Shreir, L. L. (1994), Jarman, R. A.; Brustein, G. T., Eds. Butterworth-Heinemann, Vol. 1, 1:293-1:303.

2. ASTM (2006), Standard Terminology Relating to Corrosion and Corrosion Testing. 
3. Postlethwaite, J. and Nesic, S. (2000), Uhlig's Corrosion Handbook. 2nd ed.; Reive, R. W., Ed. John Wiley \& Sons, 249-272.

4. Poulson, B. S. (1983), Electrochemical measurements in flowing solutions, Corrosion Science 23 (4), 391-430,

5. Gonzales, J. A., Benito, M. and Feliu, S. (1995), Suitability of Assessment Methods for Identifying Active and Passive Zones in Reinforced Concrete, Corrosion, 51, 145-152.

6. Heitz, E., Henkhaus, R. and Rahmel, A. (1992), Corrosion Science: an Experimental Approach, Ellis Horwood, Chichester, UK.

7. Song, H.-W. and Saraswathy, V. (2007), Corrosion Monitoring of Reinforced Concrete Structures - A Review, International Journal of Electrochemcal Science, 2 (1), 1-28.

8. Stellwag, W. B. and Wieling, N. (1986), Electrochemical Corrosion Testing. Heitz, E.; Rowlands, J. C.; Mansfeld, F., Eds. DECHEMA, Vol. 101, 17-26.

9. Truong, V. T., Lai, P., Moore, B., Muscat, R. and Russo, M. (2000), Corrosion protection of magnesium by electroactive polypyrrole/paint coatings, Synthetic Metals, 110 (1), 7-15.
10. Easin, A. B. M. (1986), Erosion-Corrosion Studies of Steel, M. Sc. Thesis, Bangladesh University of Engineering and Technology, Dhaka, Bangladesh.

11. Burstein, G. T. and Ilevbare, G. O. (1996), The effect of specimen size on the measured pitting potential of stainless steel, Corrosion Science, 38 (12), 2257-2265.

12. NACE State-of-the-Art Report on Controlledflow Laboratory Corrosion Tests (1995), NACE Publication 5A195.

13. Sasaki, K. and Burstein, G. (2007), Erosioncorrosion of stainless steel under impingement by a fluid jet. Corrosion Science, 49 (1), 92102.

14. Vargel, C. (2004), Corrosion of Aluminium, Elsevier. 